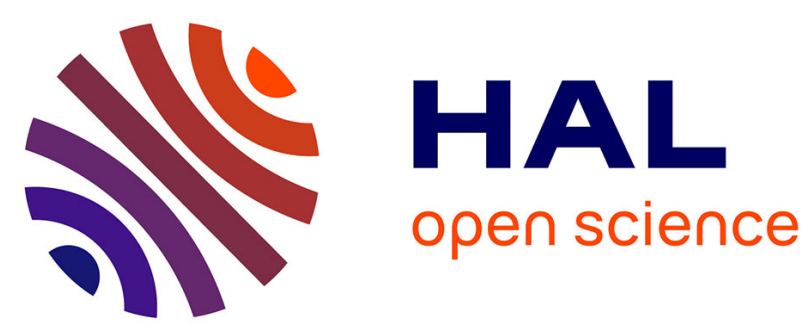

\title{
L'emploi de la bauge dans l'architecture protohistorique de Lattes (fin du VIe s.-milieu du IVe s. av. J.-C.)
}

\author{
Jean-Claude Roux
}

\section{To cite this version:}

Jean-Claude Roux. L'emploi de la bauge dans l'architecture protohistorique de Lattes (fin du VIe s.-milieu du IVe s. av. J.-C.). Gallia - Archéologie de la France antique, 2008, Lattara/Lattes (Hérault) : nouveaux acquis, nouvelles questions sur une ville portuaire protohistorique et romaine, 65, pp.107-109. 10.3406/galia.2008.3334 . hal-01913938

\section{HAL Id: hal-01913938 \\ https://hal.science/hal-01913938}

Submitted on 19 Dec 2019

HAL is a multi-disciplinary open access archive for the deposit and dissemination of scientific research documents, whether they are published or not. The documents may come from teaching and research institutions in France or abroad, or from public or private research centers.
L'archive ouverte pluridisciplinaire HAL, est destinée au dépôt et à la diffusion de documents scientifiques de niveau recherche, publiés ou non, émanant des établissements d'enseignement et de recherche français ou étrangers, des laboratoires publics ou privés.

\section{(ㅇ)(1) $\$$}

Distributed under a Creative Commons Attribution - NonCommercial - NoDerivatives $\mid 4.0$ 


\title{
L'EMPLOI DE LA BAUGE DANS L'ARCHITECTURE PROTOHISTORIQUE DE LATTES (FIN DU VI ${ }^{\mathrm{e}}$ S.-MILIEU DU IV ${ }^{\mathrm{e}}$ S. AV. J.-C.)
}

\author{
Jean-Claude Roux
}

Mots-clés. Architecture en terre crue, soubassement, bauge, matériau de construction, techniques constructives, mise en cuvre.

Résumé. Inconnu encore en Protohistoire il y a quelques années, le montage des murs d’habitation en bauge a bénéficié à Lattes d'observations multiples qui ont permis une caractérisation précise de cette technique. Principalement attesté entre la fin du VI ${ }^{e}$. et le milieu du IV s. av.J.-C., ce mode de construction présente plusieurs formes de mise en æuvve et se trouve employé souvent en même temps que d'autres techniques, utilisant la pierre ou la brique, dans la même maison, voire parfois dans un même mur.

Key-words. Clay architecture, footing, wallow, building material, building tehniques, execution.

Abstract. Still unknown for Protohistoric phases these last years, the wallow walls of dwellings have been well analysed at Lattes and it made possible a precise charaterization of this technique. Mainly attested between the end of the $6^{\text {th }}$ century and the mid $4^{\text {th }}$ century B.C., this building technique shows several kinds of execution and often is used at the same time than other techniques using stone or brick, in the same house or even in the same wall.

Translation: Isabelle FAUDUET

Schlüsselwörter. Lehmarchitektur, Sockel, Strohlehm, Baumaterial, Konstruktionstechniken, Einbringung.

Zusammenfassung. Die bis vor einigen Jahren auf frühgeschichtlichen Fundstätten noch unbekannte Verwendung von Strohlehm für die Errichtung von Wohnhausmauern konnte in Lattes eingehend untersucht und dank dieser Beobachtungen genau beschrieben werden. Die überwiegend zwischen dem ausgehenden 6. und der Mitte des 4. Jahrhunderts v.u.Z. bezeugte Konstruktionstechnik weist mehrere Formen der Materialeinbringung auf und wird oft in demselben Haus, ja sogar an derselben Mauer gleichzeitig mit anderen Techniken eingesetzt, bei denen Stein oder gebrannte Lehmziegel verwandt werden.

Übersetzung: Isa ODENHARDT-DONVEZ

Il y a une quinzaine d'années, on ignorait encore que l'habitat ancien de Lattara utilisait dans l'architecture une technique constructive par façonnage direct de terre crue : la bauge ou terre massive. Les structures en bauge apparaissent dans les niveaux inférieurs du site localisés pour les plus récents à une profondeur de 2,50 m. Ces niveaux sont la plupart du temps recouverts par la nappe phréatique, d'où la difficulté de reconnaître la terre crue façonnée. Le matériau est une terre limoneuse utilisée à l'état plastique par addition d'eau, contenant peu ou pas de fibres végétales, qui doit reposer un à deux jours avant l'emploi. La gâchée était souvent préparée à l'intérieur même des maisons. Les apports de terre se faisaient vraisemblablement par petites quantités et au fur et à mesure de leur utilisation, ce qui expliquerait pourquoi, dans un même mur, on retrouve des terres de nature différente ; la préparation peut être évaluée entre $0,50 \mathrm{~m}^{3}$ et $1 \mathrm{~m}^{3}$ par gâchée (Roux, 2003, p. 272). Sur le site de Lattes, la bauge est principalement employée dans l'architecture domestique et pour certains aménagements intérieurs comme les banquettes et les sols pavés.

Durant le $\mathrm{V}^{\mathrm{e}}$ s. av. J.-C., la bauge prédomine dans le montage du solin des murs (fig. 77). L'emploi de la pierre en 


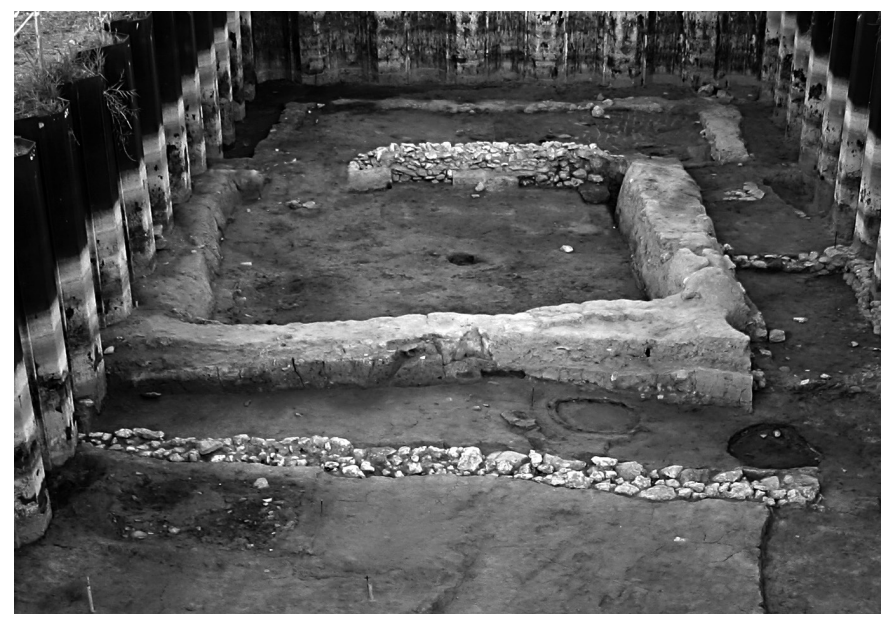

Fig. 77 - Soubassements en bauge et en pierre d'une maison du milieu $d u V^{e}$ s. av. J.-C. dans la zone 1 de Lattes (cliché: E. Gailledrat, CNRS).

soubassement se limite alors à une partie bien spécifique du bâtiment, sur un mur ou un tronçon de mur d'une maison, généralement pour renforcer le piédroit d'une ouverture ou une tête de mur. En revanche, les élévations sont le plus souvent bâties en briques crues (Roux, à paraître). Ce mode de construction se rencontre encore couramment au premier quart du IV ${ }^{\mathrm{e}}$ s. av. J.-C. dans des maisons indépendantes ou associées à d'autres maisons en pierre et adobe au sein d'un même îlot (Roux, 1999, p. 15 et p. 32-35). On assiste ensuite à un progressif abandon de cette technique, les constructions postérieures au milieu du $\mathrm{IV}^{\mathrm{e}} \mathrm{s}$. av. J.-C. utilisant désormais principalement une architecture mixte comprenant un soubassement en pierres liées à la terre surmonté d'une élévation en adobes (Chazelles, 1996, p. 259-328).

Les murs en bauge de Lattes ont été présentés et décrits dans plusieurs études archéologiques ou thématiques au fur et à mesure de leur découverte (Chazelles, 1999, p. 229-239 ; Roux, 1999, p. 15, p. 32-35 et 2003). Le référentiel actuel d'une cinquantaine de murs en bauge, mis au jour sur le site et pour la plupart fouillés finement, permet de dresser une première synthèse sur ce type de construction, sur la base d'observations de terrain et d'analyses micromorphologiques des matériaux (Cammas, 1999, p. 214-216).

Les murs en terre crue sont construits directement sur le sol grossièrement égalisé si nécessaire, sans tranchée d'ancrage ni fondation. À la base, une couche de mise à niveau (lit de pose) peut être étalée sur une épaisseur de $2 \mathrm{~cm}$ à $15 \mathrm{~cm}$ selon les anfractuosités du terrain. La superposition d'un mur en bauge sur un mur antérieur arasé, de même nature ou constitué de pierres, est fréquent du fait d'une certaine stabilité des espaces privatifs. Une structure en terre massive peut aussi être bâtie à cheval sur un sol en terre battue et les ruines d'un mur. Un seul exemplaire d'ancrage de mur fondé sur un radier de pierre a été rencontré dans l'îlot 32 et est daté de la première moitié du IV ${ }^{\mathrm{e}}$ s. av. J.-C. ; il s'agit de gros blocs calcaires disposés irrégulièrement à plat dans le sens du mur, mais qui ne couvrent cependant pas toute la longueur de la structure (Roux, 2003, p. 265 et fig. 4).

Les solins présentent une épaisseur comprise entre $0,55 \mathrm{~m}$ et $1,50 \mathrm{~m}$, avec une moyenne autour de $0,70-0,80 \mathrm{~m}$, dimensions plus importantes que pour les solins de pierre épais de $0,50 \mathrm{~m}$ en moyenne. Deux exemplaires dans la maison 124 de l'îlot 1D, datée du début du IV s. av. J.-C., offrent une base exceptionnellement large de $1,30 \mathrm{~m}$; l'élévation devait être vraisemblablement plus étroite et correspondre aux dimensions des autres murs en terre de l'îlot, comprises entre $0,60 \mathrm{~m}$ et 0,70 m. La particularité de ces deux murs est qu'ils comportent dans leur épaisseur une armature de poteaux fortement ancrés dans le sol (Roux, 1999, p. 32-34 et fig. 47).

La hauteur des soubassements est difficile à établir du fait que la partie conservée varie entre $0,10 \mathrm{~m}$ et $0,88 \mathrm{~m}$. Quelques exemplaires aboutés à des solins en pierre avec arase de tessons ont une hauteur de $0,50 \mathrm{~m}$, d'autres conservés par superposition de murs donnent un solin de $0,40 \mathrm{~m}$ de haut. Un cas de basculement d'un pan de mur en terre crue permet de reconstituer son élévation, avec une partie inférieure en bauge haute de 1,60 m surmontée par au moins $0,80 \mathrm{~m}$ de briques crues. La hauteur moyenne se situe néanmoins autour de 0,40-0,50 m.

En règle générale, le matériau de construction employé dans les soubassements en bauge lattois correspond à des terres limono-argilo-sableuses contenant différentes sortes d'agrégats. Il s'agit de terres anthropisées pouvant contenir des petits fragments de céramiques et d'os ainsi que des charbons de bois, des éléments de briques crues plus ou moins désagrégées et des limons sédimentaires. Ces matériaux sont mélangés sans ordre. La présence de charbons de bois résulte sans doute de la préparation sur le sol des habitations et peut donc être interprétée comme un mélange accidentel. La terre est parfois renforcée au façonnage par des fragments d'adobes, des cailloux et des tessons épars ou concentrés selon les endroits, qui améliorent la résistance mécanique et limitent le retrait au séchage (fig. 78, $\mathrm{n}^{\mathrm{o}} 3$ ).

Quatre formes de mise en œuvre ont été individualisées jusqu'à présent. Le mode le plus courant consiste à superposer des lits de terre à l'état de pâte cohérente humide 
semi-plastique, d'une épaisseur inégale de $10 \mathrm{~cm}$ à $20 \mathrm{~cm}$, s'interpénétrant sur la hauteur ou horizontalement selon le façonnage. Ces couches, souvent de texture et de nature diverses, ne sont pas obligatoirement disposées sur toute la largeur de la structure. Elles peuvent se répartir sur un segment de mur ou s'étaler sur deux tronçons (chaînage) au fur et à mesure de l'avancement de la construction. Des boules de terre peuvent être incluses dans le façonnage. Cette technique de terre litée implique un minimum de séchage du support, ce qui suggère que dans la construction d'une maison, l'élévation des murs pouvait s'effectuer en tournant (fig. $78, \mathrm{n}^{\text {os }} 1$ à 3 ).

Le deuxième mode, de loin le moins utilisé, consiste à amalgamer des boules de terre et/ou à juxtaposer des mottes de terre à l'état plastique, côte à côte, dans l'axe ou sur toute la largeur du mur (fig. 78, $n^{\circ} 5$ ).

La troisième technique, plus rare, consiste à maintenir de la terre préparée entre deux parois latérales modelées en terre. Deux manières de faire ont été reconnues, toujours à la base des murs : la première correspond à des parois irrégulières de $15 \mathrm{~cm}$ à $25 \mathrm{~cm}$ d'épaisseur, façonnées de part et d'autre de la structure sur une trentaine de centimètres de haut, entre lesquelles est déposée une couche de terre (fig. 78, no 4) ; la seconde consiste à façonner deux bourrelets triangulaires présentant une face extérieure verticale et une face intérieure oblique à $45^{\circ}$, le centre étant rempli de terre préparée (fig. $78, \mathrm{n}^{\circ} 1$ ). Cette disposition crée un berceau en $\mathrm{V}$ qui annule quasiment les poussées latérales et réduit les risques de glissement de la base du mur sous le poids de l'élévation. Ce procédé est bien attesté dans les murs et le bouchage de la porte d'une habitation du troisième quart du $\mathrm{V}^{\mathrm{e}} \mathrm{s}$. de la zone 1 (maison 132) et dans trois autres murs plus récents d'un demi-siècle, mais il reste peu commun et semble réservé pour des utilisations spécifiques (Roux, à paraître).

Le quatrième mode de construction concerne la bauge coffrée, identifiée notamment dans le mur d'une maison du début du IV é s. de la zone 27 (Roux, Cammas, 2007).

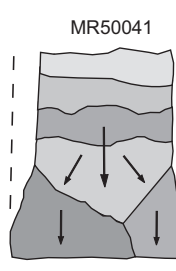

1

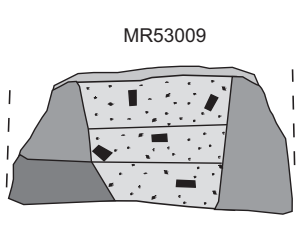

4

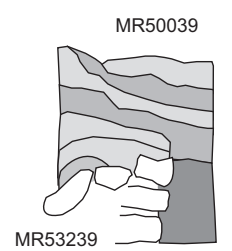

2

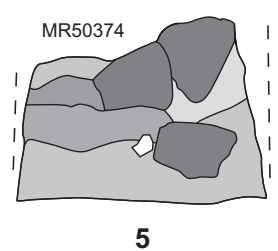

$1 \mathrm{~m}$

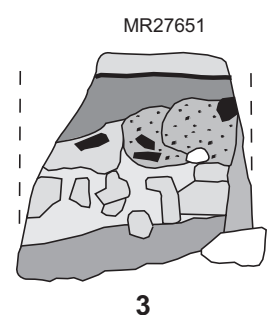

MR27717

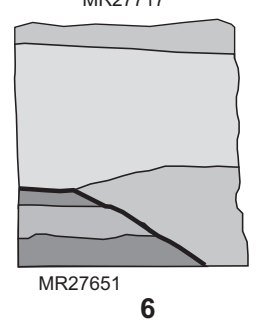

Fig. 78 - Différents types de mise en œuvre des murs en bauge de Lattes : 1 , bourrelets à $45^{\circ}$ surmontés de couches litées; 2 , couches litées; 3, couches litées mêlées de boules de terre, de fragments d'adobes et de tessons; 4 , parois de coffrage; 5 , mottes de terre; 6 , bauge coffrée (DAO : J.-C. Roux, SRA Languedoc-Roussillon).

Ce mur se compose de trois levées de terre très compactes et homogènes, parfaitement uniformes dans l'ensemble de la structure, les deux premières présentant une hauteur moyenne de 0,40 m (fig. 78, $\mathrm{n}^{\circ} 6$ ). L'utilisation de coffrages en bois est probablement sous-estimée sur le site de Lattes, les analyses micromorphologiques montrant souvent l'emploi d'une terre dans un état boueux ou saturé en eau, nécessitant un coffrage lors de la construction (Cammas, 1999, p. 215-216 et tabl. 4).

La fouille de soubassements en bauge, relativement bien conservés dans les niveaux anciens de Lattara, apporte donc des données précises et originales sur les modes d'utilisation de la terre dans l'architecture protohistorique méditerranéenne et permet de préciser l'évolution affectant, au cours de l'âge du Fer, les techniques de mise en œuvre de ce matériau. 